\title{
Spinal cord compression due to carcinoid metastasis
}

\author{
J.A. Gray, ${ }^{1}$ H. Nishikawa, ${ }^{2}$ M.A. Jamous ${ }^{2}$ and D.G. Grahame-Smith ${ }^{1}$ \\ ${ }^{1}$ MRC Unit and University Department of Clinical Pharmacology, Radcliffe Infirmary, Oxford and \\ ${ }^{2}$ Department of Neurosurgery, Radcliffe Infirmary, Oxford, UK.
}

\begin{abstract}
Summary: A case of cord compression secondary to the carcinoid syndrome is reported. The patient made an excellent response to surgical treatment, emphasizing that active management of this unusual complication should be considered.
\end{abstract}

\section{Introduction}

Carcinoid tumours only rarely metastasize to the central nervous system. ${ }^{1}$ Secondary spinal metastatic carcinoid was first described by Kirkpatrick et al. ${ }^{2}$ while Manoli and Barthelemy ${ }^{3}$ were the first to report cord compression due to bony metastases.

A case of spinal cord compression due to cervical vertebral metastases is presented as well as followup details on a previously reported patient from our hospital. ${ }^{4}$ Both patients initially responded well to treatment emphasizing the importance of early recognition and active management of this condition.

\section{Case report}

A 63 year old female presented originally 8 years previously with a 7-year history of flushing attacks aggravated by alcohol ingestion and a 2-year history of intermittent diarrhoea. Examination at the time of her initial presentation revealed a hard enlarged liver and signs of tricuspid regurgitation and pulmonary stenosis. She also had generalized pigmentation and thickening of the skin especially of the hands and forearms (pseudoscleroderma). Urinary 5-hydroxyindole-acetic acid (5-HIAA) level was greatly elevated, confirming the diagnosis of carcinoid syndrome. Urine chromatography showed that in addition to 5-HIAA, 5-hydroxytryptamine (5-HT) and 5-hydroxytryptophan were also present in excess, suggesting that the tumour was poorly differentiated and perhaps bronchial or pancreatic in origin. ${ }^{1}$ The whole blood 5-HT concentration was almost twice the normal value $(586 \mathrm{ng} / \mathrm{ml}$; normal value up to $300 \mathrm{ng} / \mathrm{ml}$ ). Unusually the whole blood histamine level was also greatly elevated.

Correspondence: J.A. Gray, M.A., M.R.C.P. (UK)

Accepted: 4 May 1988
Radiological investigation of the chest and bowel ํㅝ failed to reveal the primary site of the tumour. She made a good response to medical treatment with $\omega$ methysergide and cyproheptadine but developed a 을 carcinoid crisis due to necrosis of hepatic secondar- ies 4 years later. She underwent partial hepatectomy $\mathscr{D}$ which led to great symptomatic improvement.

On the present occasion she presented with a 3week history of increasing weakness of the arms and legs, such that she had been unable to walk fof 2 days.

On examination she had weakness of the legs with brisk knee and ankle reflexes and extens plantar responses. There was weakness and wasting of the deltoid muscles especially on the left. The biceps and supinator reflexes were absent but the triceps reflexes were brisk.

$X$-ray of the cervical spine confirmed the presence of a destructive lesion of the fifth cervical vertebra involving the vertebral body with dislocation of $\mathrm{C} 4 / 5$ (Figure 1).

She was placed on Halo skull traction and was started on dexamethasone, at a dose of $4 \mathrm{mg}$ four times a day. Power in her limbs returned to normal within $48 \mathrm{~h}$; check cervical X-ray showed realignment of the cervical spine. The Halo cervical traction was converted into a Halo vest and she was mobilized. A subsequent course of radiotherapy (total dose $3000 \mathrm{rad}$ ) failed to induce fusion in the cervical spine after 6 weeks. Consequently, it $\frac{7}{0}$ was felt that resection of the body of $\mathrm{C} 5$ and fusion was the only remaining option. This was performed $N$ through an anterior approach: the body of C5 was $\mathrm{N}$ resected and replaced with acrylic, and fusion from C4 to C6 was carried out. Histology confirmed the presence of carcinoid tumour in the excised bone.

She was able to return home and remained reasonably well and mobile until 6 months later $\mathbb{Q}$ when she presented with a skull mass. A skull X-? ray confirmed secondary spread to the skull with

(C) The Fellowship of Postgraduate Medicine, 1988 


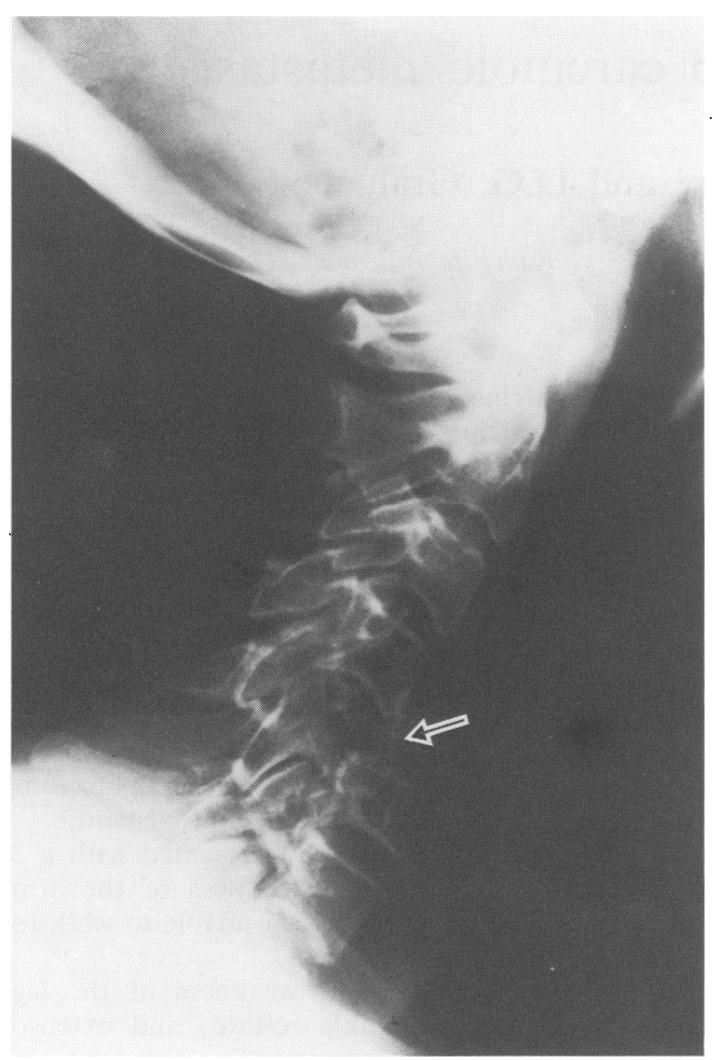

Figure 1 Radiograph of the cervical spine showing destruction and dislocation of the fifth cervical vertebra by carcinoid tumour.

lytic lesions. No further active management was felt appropriate at this point. One month later she slipped down a flight of stairs sustaining a blow to the back of her head. She did not lose consciousness at the time of the injury but over the subsequent 4 hours complained of headache, drowsiness and increasing difficulty in walking. She was admitted to hospital where 2 hours later she suddenly became unconscious with a fixed dilated left pupil and lack of response to painful stimuli. Computed tomographic scan showed a large extradural mass involving the skull, compressing both frontal lobes; this was assumed to be a metastatic deposit. She died 3 days later.

\section{Discussion}

This case illustrates how carcinoid metastases may lead to cord compression by causing vertebral destruction. The osteolytic nature of the vertebral secondary in our case is unusual, most bony metastases reported being osteoblastic in nature. ${ }^{3}$ Furthermore while there have been five previous reports of cord compression secondary to carcinoid tumour $^{2-6}$ in all but one ${ }^{3}$ of these the cord appears to have been compressed by direct spread rather than by bony destruction. The spinal cord is most commonly compressed at the thoracic or lumbar regions although a case of cord compression in the cervical region has been previously reported. ${ }^{3}$

This case was also unusual in that the skull became involved with intracranial extension leading to raised intracranial pressure which was an important factor in her death. The radiological evidence suggested that the tumour was compressing rather than invading the brain tissue as indicated by the lack of cerebral oedema which would be expected in the latter case. There is no published evidence of similar cases although direct metastatic spread to the brain has been reported on five occasions. ${ }^{7-11}$

A further case of spinal cord involvement due to carcinoid metastasis was reported previously from this hospital. ${ }^{4}$ The patient presented with a cauda equina syndrome, with weakness and wasting of both thighs and the right buttock due to a sclerotic deposit in the second lumbar vertebra. He underwent decompressive lumbar laminectomy followed by a course of radiotherapy and made a full recovery. He remained well for 3 years after his surgery but subsequently was readmitted with further evidence of cord compression. Despite further surgery he died from pneumonia 1 month later.

There are no clear data available concerning the sensitivity of carcinoid tumours to radiotherapy. In our case there was no response in terms of inducing bony fusion by radiotherapy. However the response to surgery was excellent, leading to symptomatic and functional improvement. Although the patient died from multiple metastases within 1 year of surgery, her quality of life up to the time of death was good. Since the patient had already responded well to previous 'aggressive' surgery to the liver, the case also illustrates how sometimes a patient may live for years in symbiosis with a carcinoid tumour, even after metastasis has occurred.

The prognosis for life in many cases of the carcinoid syndrome is long, particularly those with 'typical' metastatic ileal carcinoids. For this reason, and because of the potentially excellent therapeutic response, spinal cord compression due to carcinoid metastases should be treated surgically, with or without radiotherapy, if the overall clinical condition makes this appropriate. 


\section{References}

1. Grahame-Smith, D.G. Carcinoid Syndrome. Heinemann, London, 1972.

2. Kirkpatrick, D.G., Dawson, E., Haskell, C.M. \& Batzdorf, U. Metastatic carcinoid presenting as a spinal tumour. Surg Neurol 1975, 4: 283-287.

3. Manoli, B.S. \& Barthelemy, C.R. Osteolytic and osteoblastic metastases due to carcinoid tumours. Clin Nucl Med 1980. 5: 102-105.

4. Rawlinson, W.A.L., Marsh, R.H.K. \& Goat, V.A. Anaesthesia for removal of carcinoid metastases. Anaesthesia 1980, 35: 585-588.

5. Ansink, B.J.J., Van Lambalgen, H.C.E. \& Davies, G. Metastatic carcinoid tumour compressing the cauda equina. J Neurol Neurosurg Psychiat 1984, 47: 652 (letter).

6. Gowitt \& Mirra. Malignant carcinoid causing spinal cord compression. Neurosurgery 1985, 17: 801-806.
7. Karkos, J. A morphologic picture of the iliac carcinoid metastasis to the cerebellum. Neuropatol Pol 1971, 9: 231-237.

8. Wagner, P.K., Gabbert, H. \& Brunner, H. Gastric carcinoid with brain metastases. Med Klin 1979, 74: 1439-1441.

9. Robertson, M.G. Carcinoid tumour. Arch Intern Med 1983, 143: 389-390.

10. Bouldin, T.W., Killebrew, K., Boone, S.C. \& Gay, R.M. Metastasis of a rectal carcinoid to the posterior fossa. Neurosurgery 1979, 5: 496-499.

11. Schiffman, F.J. \& Bernard N.J. Carcinoid tumour of the gastrointestinal tract with metastases in the brain. Arch Intern Med 1982, 142: 1717-1719. 